\title{
Early Phase in the Development of Cannabidiol as a Treatment for Addiction: Opioid Relapse Takes Initial Center Stage
}

\author{
Yasmin L. Hurd ${ }^{1}$ - Michelle Yoon ${ }^{1} \cdot$ Alex F. Manini $^{2} \cdot$ Stephanie Hernandez $^{2}$ • \\ Ruben Olmedo $^{2} \cdot$ Maria Ostman $^{3} \cdot$ Didier Jutras-Aswad ${ }^{4}$
}

Published online: 13 August 2015

(C) The American Society for Experimental NeuroTherapeutics, Inc. 2015

\begin{abstract}
Multiple cannabinoids derived from the marijuana plant have potential therapeutic benefits but most have not been well investigated, despite the widespread legalization of medical marijuana in the USA and other countries. Therapeutic indications will depend on determinations as to which of the multiple cannabinoids, and other biologically active chemicals that are present in the marijuana plant, can be developed to treat specific symptoms and/or diseases. Such insights are particularly critical for addiction disorders, where different phytocannabinoids appear to induce opposing actions that can confound the development of treatment interventions. Whereas $\Delta^{9}$-tetracannabinol has been well documented to be rewarding and to enhance sensitivity to other drugs, cannabidiol (CBD), in contrast, appears to have low reinforcing properties with limited abuse potential and to inhibit drug-seeking behavior. Other considerations such as CBD's anxiolytic properties and minimal adverse side effects also support its potential viability as a treatment option for a variety of symptoms associated with drug addiction. However, significant research is still needed as CBD investigations published to date primarily relate to its effects on opioid drugs,
\end{abstract}

Yasmin L. Hurd

yasmin.hurd@mssm.edu

1 Departments of Psychiatry, Neuroscience and Pharmacology and Systems Therapeutics, Icahn School of Medicine at Mount Sinai, New York, NY, USA

2 Division of Medical Toxicology, Department of Emergency Medicine, Icahn School of Medicine at Mount Sinai, New York, NY, USA

3 Department of Psychiatry, Karolinska Institutet, Stockholm, Sweden

4 Research Center, Centre Hospitalier de l'Université de Montréal, Department of Psychiatry, Université de Montréal, Montreal, Canada and CBD's efficacy at different phases of the abuse cycle for different classes of addictive substances remain largely understudied. Our paper provides an overview of preclinical animal and human clinical investigations, and presents preliminary clinical data that collectively sets a strong foundation in support of the further exploration of CBD as a therapeutic intervention against opioid relapse. As the legal landscape for medical marijuana unfolds, it is important to distinguish it from "medical CBD" and other specific cannabinoids, that can more appropriately be used to maximize the medicinal potential of the marijuana plant.

Keywords THC $\cdot$ Cannabis $\cdot$ Heroin $\cdot$ Human $\cdot$ Rat $\cdot$ Craving

\section{Introduction}

With debates about so-called medical marijuana and the widespread media coverage on the subject, the call for the legalization of marijuana (Cannabis sativa) both for recreational and medical purposes has gained considerable momentum in recent years. While much attention has been given to the medicinal promises that the marijuana plant might possess, the spotlight on marijuana has also raised awareness about the remarkable dearth of scientific studies that have been conducted on this plant's therapeutic potential. As shown in Fig. 1, the number of research studies published on cannabis has coincided temporally with major changes in the social and political climates of the time such as in the early 2000s after states such as California legalized marijuana. Unfortunately, many scientific and medical questions remain with respect to the potential or actual benefits and risks of medicinal and recreational marijuana use. Although the public and the media use the term "medical marijuana" liberally, few acknowledge or are even aware of the complex nature of the plant, which consists 


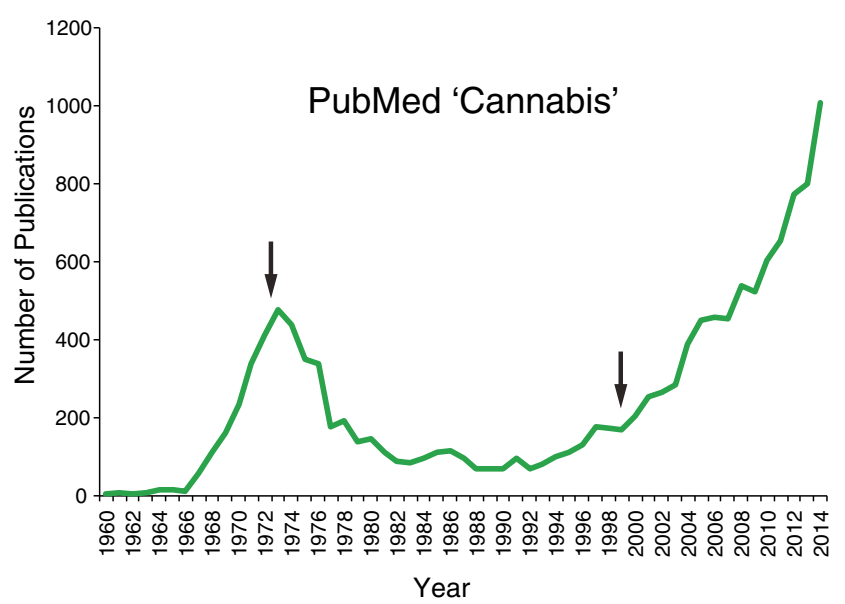

Fig. 1 The number of publications based on PubMed search for the term "cannabis". Patterns coincides with governmental policy and societal changes (some denoted by arrows), such as cannabis becoming a Schedule I drug in the USA in 1970, in the 1970s state laws and local regulations begin to ban possession or sale of cannabis, in 1996 California voters passed Proposition 215 that legalized medical cannabis, and in 2000 there were increased attempts for decriminalization and legalized marijuana use around the USA. No other cannabinoid-related PubMed search term showed the same temporal pattern

of $>400$ chemicals, with approximately 70 cannabinoids $[1,2]$. The truth is, there is growing evidence that not all components of marijuana are medically beneficial and it is still unclear as to what specific medical disorders are best treated by this plant. Which cannabinoids mediate what specific beneficial or adverse effects remains an important question when one considers the complexity of the marijuana plant, and there is now growing research interest in answering such questions in the hopes of identifying and developing medicinal cannabinoids targeted for specific medical symptoms and diseases.

Most scientific studies to date have focused on $\Delta^{9}$-tetrahydrocannabinol (THC), the most prominent psychoactive constituent of the plant and the cannabinoid that leads to the rewarding effects of cannabis. Another prominent phytocannabinoid is cannabidiol (CBD) [2], which has extremely low concentrations in the marijuana strains commonly used recreationally in which the THC potency has dramatically increased [3]. Plants more recently cultivated with a high CBD content (and low THC levels) are thought to have potential benefit in treating various diseases; in particular, CBD as an antiepileptic agent in children has received much public interest, even though the evidence to date has been mostly anecdotal, with active clinical trials now underway $[4,5]$. In this article, we focus on CBD's potential therapeutic potential in addiction disorders based on evidence from preclinical studies that suggest a "yin/yang" relationship between 2 components of the cannabis plant where one enhances substance abuse risk (THC) and the other inhibits drug relapse (CBD) (Fig. 2). The information below provides a foundation for the development of CBD as a potential treatment for addiction to specific drug classes.

\section{Overview of Addiction and Current Treatment Challenges}

Individuals who suffer from addictive disorders go through various stages in their illnesses (Fig. 3), each of which is characterized by specific neurobiological states. Substance use and intoxication produce psychoactive and addictive effects by acting on the brain's reward system - a set of interconnected regions that control pleasure and motivation [6]. During the intoxication phase, drugs modulate a number of neurotransmission systems such as dopamine, opioid, serotonin, and norepinephrine. These biochemical events are responsible for the physiological and behavioral effects observed in abusers (e.g., euphoria, restlessness, and tachycardia). Early abstinence in dependent patients results in pharmacological and clinical effects that are opposite to those found during the intoxication phase. Acute withdrawal may include symptoms that vary according to the type of drug, including
Fig. $2 \Delta^{9}$-tetrahydrocannabinol (THC) and cannabidiol (CBD) have opposing "yin/yang" effects on addiction-related behaviors. In contrast to THC that is rewarding and promotes drug use, CBD has low hedonic property and inhibits drug seeking

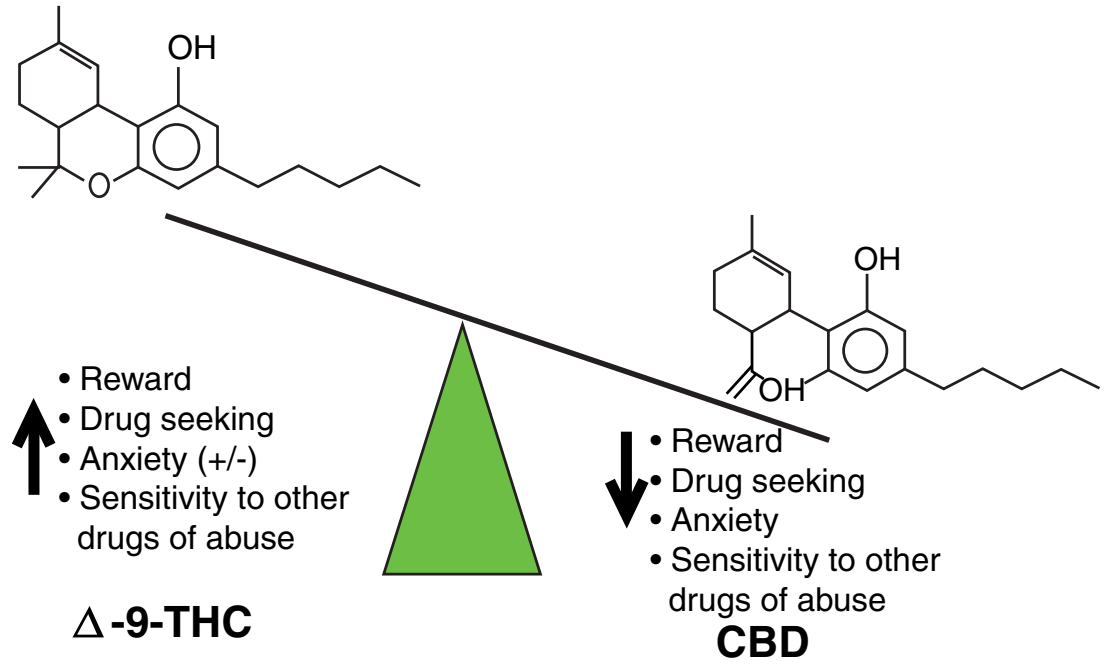


sleep disturbances, anxiety, dysphoria, and fatigue [7]. Most importantly, this phase is associated with low stress tolerance and recurrent episodes of craving (an intense desire to use), which persist for months, often resulting in relapse [8, 9]. In the long term, addiction becomes characterized by compulsive substance use and, most strikingly, repetitive urges to consume the drug can persist even after sustained periods of abstinence.

While substance use remains the most obvious direct outcome of addiction, there is now growing interest among scientists to focus on other core symptoms of this disorder. In the recently published Diagnostic and Statistical Manual of Mental Disorders, 5th Edition, craving - the most prominent symptom and long-lasting sequel of drug dependence-has been added to the criteria of substance use disorders, a direct reflection of its clinical relevance in addictive disorders. Craving has become a subject of great interest as it is a reliable intermediate phenotype of relapse and the most distressing and long-lasting symptom experienced by dependent individuals between uses. Indeed, even after a period of abstinence, dependent individuals remain vulnerable to stress and other craving-inducing stimuli [10], which, in turn, leads to intense physiological responses and various negative feelings such as anger and sadness [11]. Real-time daily monitoring of craving and drug use has shown that craving reliably predicts relapse among dependent individuals [9, 12-15]. The data suggest that improving the treatment of craving could not only help prevent relapse, but could also reduce patient distress on the emotional, cognitive, and physiological levels.

While significant scientific efforts have been deployed over the last few decades in the development of interventions that target craving and different phases of the addiction cycle (Fig. 3), their success rates have been limited. Psychosocial approaches have been widely used to help patients achieve improved outcomes after drug cessation; however, the literature indicates that these strategies alone are at times insufficient to induce significant behavioral changes or a reduction in rates of drug consumption [16]. In addition, most of the available medication for treating addiction (e.g., alcohol and nicotine dependence) have had low-to-moderate effects on relapse outcomes. Even more concerning is the fact that no pharmacological treatment for substance abuse have yet been proven completely effective in preventing relapse for a number of substances including cocaine, amphetamine, and cannabis. The treatment options that have been explored, which include trials of medications known to regulate monoamine neurotransmission such as antidepressants, anticonvulsants, and antipsychotics, have been the subject of several systematic reviews but they have not demonstrated their efficacy in improving outcomes [17-19]. Although an immunotherapeutic vaccine strategy to hinder the passage of drugs through the blood-brain barrier is currently underway, its efficacy and realistic implementation appear unclear thus far [20,21]. With these developments and challenges in mind, a rightful sense of urgency persists within the scientific community for the identification of new compounds that will help patients initiate abstinence and avoid relapse.

\section{The Endocannabinoid System as a Treatment Target for Addiction}

Among potential emerging neurobiological targets for treating craving and addiction, the endogenous cannabinoid receptors and ligands that constitute the endocannabinoid (eCB) system have been the subject of growing interest. The eCB has tight neurobiological interaction with other neurotransmission systems that have important implications for the neural adaptations induced by drug use. For example, type 1 cannabinoid receptors $\left(\mathrm{CB}_{1} \mathrm{R}\right)$ are co-localized with opioid $\mu$ opioid
Fig. 3 Cycle of addiction and currently available medications for substance use disorders at each stage of the cycle
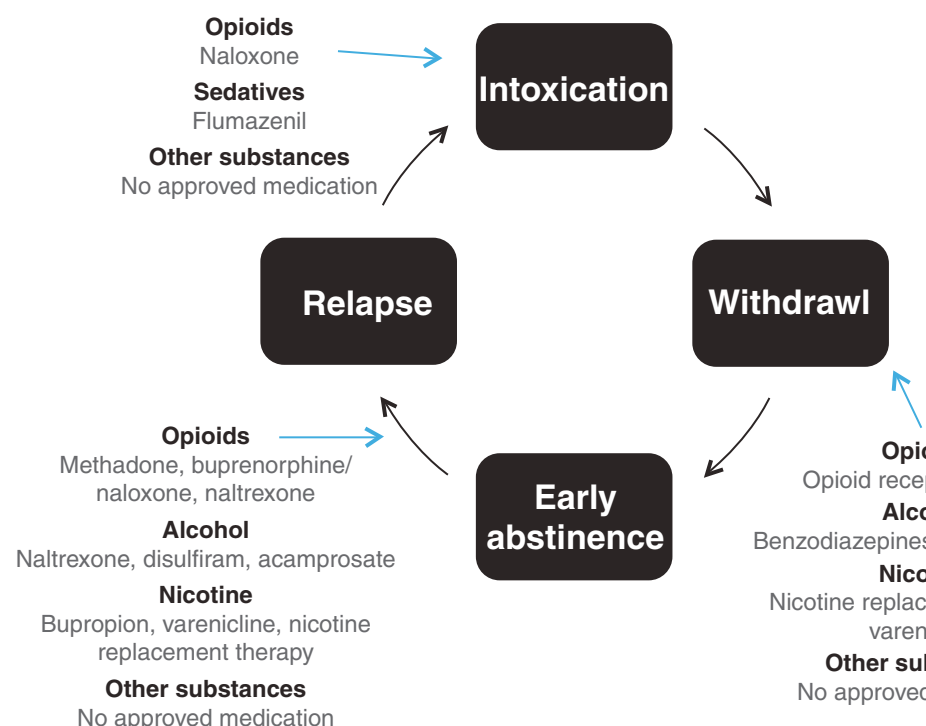

Early abstinence

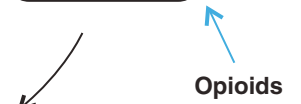

Opioid receptor agonist Alcohol

Benzodiazepines, anticonvulsant Nicotine Nicotine replacement therapy, varenicline

Other substances

No approved medication 
receptors (which mediate the actions of opioid drugs) in striatal output projection neurons of the nucleus accumbens and dorsal striatum that modulate reward, goal-directed behavior, and habit formation relevant to addiction [22]. Type 2 cannabinoid receptors $\left(\mathrm{CB}_{2} \mathrm{R}\right)$ have very low expression in the brain generally, but recently they have been shown to be expressed in dopamine neurons of the midbrain ventral tegmental area and modulate the functional excitability of dopamine neurons central to addiction related behaviors such as drug reinforcement [23]. Stimulation of $\mathrm{CB}_{2} \mathrm{R}$ in mice models has an inhibitory influence on cocaine and alcohol selfadministration and related conditioned place preference, as well as nicotine place preference behavior [23, 24].

\section{CBD and Neurobiological Targets/Effects}

Interestingly, different cannabinoids that target the eCB system exhibit distinct properties on addictive behavior. It is generally well known that THC, the predominant psychoactive cannabinoid in the cannabis plant, has high affinity at the $\mathrm{CB}_{1} \mathrm{R}$, where it acts as a partial agonist to elicit potent rewarding effects. We focus this review on CBD as it is a cannabinoid that has not been extensively studied to date and is currently being explored for its potential antiaddiction properties. CBD has long been recognized as a nonpsychotropic constituent of cannabis and is generally the second most abundant cannabinoid present in the plant $[2,3]$. Contrary to previous beliefs that CBD did not bind directly to cannabinoid receptors, recent findings indicate that $\mathrm{CBD}$ acts as an inverse agonist at $\mathrm{CB}_{1} \mathrm{R}$ and $\mathrm{CB}_{2} \mathrm{R}[25,26]$. CBD stimulates the transient receptor potential vanilloid $1 / 2$ proteins [27], which serve as socalled ionotropic cannabinoid receptors. In addition, CBD inhibits fatty acid amide hydrolase, a catabolic enzyme that alters the hydrolysis of the endogenous cannabinoid neurotransmitter anandamide [28]. Perhaps the largest body of evidence pertains to the modulation and activation of 5 hydroxytryptamine 1A serotoninergic receptors [29-35]. CBD also has low potency for inhibiting the uptake of striatal dopamine [36]; it modulates allosterically $\mu$ and $\delta$ opioid receptors [37] and enhances adenosine signaling through uptake inhibition [36, 38]. Although more studies are needed to further understand the impact of CBD on glutamatergic neurotransmission, its protective effects on glutamate toxicity and its pharmacologic interaction with ketamine $[39,40]$, an $\mathrm{N}$ methyl-D-aspartate receptor (NMDA) antagonist, are also well documented.

By virtue of its 5-hydroxytryptamine $1 \mathrm{~A}$ receptor-modulating properties, $\mathrm{CBD}$ consistently decreases stress vulnerability and exhibits anxiolytic-like effects [29, 32-34, 41-44]. Indeed, CBD's antianxiety properties have been substantiated by elevated plus-maze and rat Vogel conflict tests [41-43]. The reduction of fear-related behaviors evoked by the prey/ predator paradigm also suggests some panicolytic properties [45]. CBD improves performance in numerous animal models of cognitive impairments [30, 46-48]. It acts as an antidepressant in animal models of depression and decreases compulsive behaviors in rodents $[35,49]$. These actions are hypothesized to be linked to CB1-related mechanisms [50, 51]. CBD has also been proven to be protective against a number of druginduced adverse outcomes in animals. For example, CBD was shown to prevent cocaine-induced hepatotoxicity [52], reverse binge ethanol-induced neurotoxicity [53], and even mitigate the cardiac effects of THC $[54,55]$. In addition, CBD administration is known to attenuate amphetamine-induced hyperlocomotion [56].

Human studies on CBD corroborate preclinical findings on its therapeutic effects on nausea, inflammation, and cerebral ischemia. CBD also possesses antipsychotic properties [2, 57-61]. Not surprisingly, and as witnessed in the aforementioned preclinical data, CBD has been shown to reduce anxiety in patients with social phobia and generalized social anxiety disorders [62-64]. CBD decreases autonomic arousal and subjective anxiety [65]; these anxiolytic effects were found to be linked to the modulation of limbic and paralimbic structures $[57,62]$. It remains to be determined if these properties translate in the attenuation of symptoms for other anxiety disorders than social phobia (e.g., post-traumatic stress disorder, panic disorder) [66]. There are contradictory results as to CBD's effect on sleep (similar to results from animal studies) as it has been associated with both wake-inducing and hypnotic properties in humans $[11,67,68]$. Altogether, many pharmacological, preclinical, and clinical properties (e.g., antipsychotic, anxiolytic) of CBD that had been demonstrated over roughly the last decade all point towards a potential role for CBD in alleviating behaviors relevant to addiction disorder. As described below, recent animal and human studies have provided supporting evidence that these properties do, indeed, translate into the modulation of addiction-related outcomes.

\section{CBD in Preclinical Addiction Models}

An important consideration in the development of any new antiaddiction medication is its relative abuse liability, which, ideally, should be low. Different animal models have confirmed the low psychotropic nature of CBD [69-71], suggesting that in contrast to what is normally observed for THC, CBD does not have hedonic property on its own, that is, it is not rewarding and does not induce drug-seeking behavior. Such studies have demonstrated that CBD does not promote conditioned place preference $[69,70]$ or increase the reinforcing efficacy of brain stimulation [71], which are both definitive characteristics of addictive substances. 
Our own research efforts have emphasized CBD's low capacity to potentiate the rewarding effects of other addictive drugs. We specifically focused on evaluating CBD's effects in relation to opioids as multiple lines of our research had already established that THC potentiates heroin selfadministration in rats controlling their own drug intake, whereas the question as to whether other cannabinoids in the cannabis plant also exhibited similar properties remained unanswered. Our results showed that repeated CBD administration $(5-20 \mathrm{mg} / \mathrm{kg})$ did not alter heroin self-administration, but clearly inhibited cue-induced heroin-seeking behavior [72]; Fig. 4). Intriguingly, CBD's effects were prolonged, lasting two or more weeks after administration in its efficacy to reduce heroin reinstatement behavior triggered by drug-specific environmental cues. Moreover, even when administered during active heroin intake, the ability of CBD to inhibit relapse behavior was still apparent weeks after the last exposure, suggesting that CBD could impact the course of heroin dependence even following a potential lapse condition after a period of abstinence. This highlights a property unique to $\mathrm{CBD}$, one that is not found in the medications currently used for the treatment of heroin abuse. Importantly, no physical side effects were noted in the animals with respect to gross effects on motor function.

Research is currently ongoing to delineate the neurobiological mechanisms by which CBD mediates its long-term effects on heroin-seeking behavior, but initial data suggest that CBD normalizes heroin-induced impairment on the $\alpha$-amino-3-hydroxy-5-methyl-4-isoxazolepropionic acid glutamate receptor (AMPA) GluR1, as well as the $\mathrm{CB}_{1} \mathrm{R}$ expression within the nucleus accumbens [72]. The glutamatergic system and particularly GluR1 receptors are known to contribute to

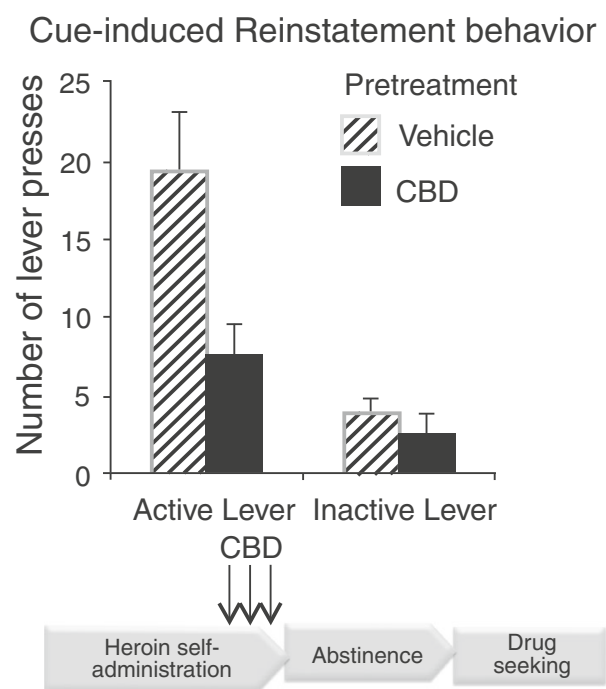

Fig. 4 Cannabidiol (CBD) effects on heroin self-administration and cue-induced heroin reinstatement behavior in rats showing CBD reduces heroin reinstatement behavior. Modified from Ren et al. [72] neuroplasticity underlying drug-seeking behavior [67, 73], and treatments targeting glutamates are being developed for addiction intervention.

Corroborating our own investigations, other animal studies have also suggested beneficial effects of CBD in relation to clinical symptoms associated with opioid exposure. One line of evidence particularly relevant to opioid abuse is the consistent findings that 1) CBD reduces morphine withdrawal symptoms (e.g., wet shakes, diarrhea, abnormal posture, ptosis, chewing, or teeth chattering) [74-77], and 2) even in combination with THC, CBD is capable of reducing abstinence scores to a greater extent than THC alone [74, 75].

Although significant preclinical animal data are accumulating with regard to $\mathrm{CBD}$ and opioid drugs, information regarding CBD and its effects on other substances of abuse are currently still very limited. Findings to date suggest minimal CBD impact on apparent positive subjective effects induced by psychostimulants or THC [70, 71, 78]. Moreover, no animal studies have been published to date regarding CBD's effects on nicotine or alcohol. Clearly, more research is needed. Importantly, most studies have predominantly evaluated CBD in models designed to only assess its immediate actions on other drugs, and have yet to fully evaluate its potential protracted effects on drug-seeking behavior and withdrawal symptoms. Of note, Parker et al. [69] found that CBD potentiated the extinction of cocaine and amphetamine-induced conditioned place preference learning, but had no impact on the establishment of conditioned place preference. The implementation of studies using animal models of relapse will be critical to inform human investigations considering the possibility of CBD as a long-lasting therapeutic agent for addiction.

\section{CBD and Human Translational Studies}

Human studies regarding CBD's potential impact on the abuse of other drugs are even more limited than preclinical animal investigations. Thus far, there has only been 1 report with cigarette-dependent participants, and CBD was observed to reduce the number of cigarettes consumed by active users[79]. The same investigative team has also evaluated CBD in relation to cannabis abuse. In naturalistic studies conducted with cannabis users, the concentration of CBD in smoked cannabis did not attenuate psychomimetic symptoms in participants when they were acutely intoxicated [80]; however, CBD reduced "wanting" and "liking" of cannabis-related stimuli [81]. Additionally, a case report in 1 patient indicated that CBD might reduce withdrawal symptoms and the amount of cannabis smoked upon resumption of cannabis use, but no systematic study has been conducted in relation to CBD and cannabis relapse behavior.

Based on the animal data supporting the effect of CBD on opioid-seeking behavior, we initiated pilot human clinical 
laboratory studies to begin to explore the potential of this cannabinoid as a medication for opioid craving. A critical first step was to document that $\mathrm{CBD}$, if combined with a potent opioid, would be safe as there was always the chance for a lapse in abstinent heroin abusers. Our double-blind, placebocontrolled cross-over phase I study in healthy subjects demonstrated that CBD (400 mg and $800 \mathrm{mg}$; approximately 10$15 \mathrm{mg} / \mathrm{kg}$ ) co-administered with intravenous fentanyl is well tolerated and does not exacerbate adverse effects associated with intravenous fentanyl administration such as respiratory depression or cardiovascular complications [82]. Measurements of CBD plasma levels showed the time to peak CBD concentration occurred at $3 \mathrm{~h}\left(\mathrm{C}_{\max } 181.2 \pm 39.8 \mu \mathrm{g} / \mathrm{l}\right.$ and $221.1 \pm 35.6 \mu \mathrm{g} / \mathrm{l}$, respectively, for the 400 -and 800 -mg doses; Fig. 5). Exploratory analysis of subjective measures scales [Positive and Negative Affect Schedule and Opioid Visual Analog Scale], as well as anxiety visual analog scale (VAS) scores suggested that CBD at the doses examined did not significantly alter their affective states, which was consistent with other reports [83].

The next pilot phase was to evaluate $\mathrm{CBD}$ as a potential treatment for heroin craving by assessing its effects in heroin abusers. A small double-blind design was conducted in opioid-dependent individuals (no dependence on any other drug than heroin according to the Diagnostic and Statistical Manual of Mental Disorders, 4th Edition) who, based on urine toxicology screening and the Clinical Opioid Withdrawal Scale, had been abstinent for at least 7 days. As the preclinical animal study had evaluated CBD effects following the administration of a single dose of CBD for 3 consecutive days [72], the human study design for the pilot experiment also had a similar design. As such, individuals were randomized to 3 consecutive days of CBD or placebo treatment before participating in laboratory sessions. The craving paradigm consisted of cueinduced craving test sessions where opioid-related and neutral video cues were presented at $1 \mathrm{~h}$ after a single $\mathrm{CBD} /$ placebo administration, $24 \mathrm{~h}$ after a single $\mathrm{CBD} /$ placebo administration, and 7 days following the final $\mathrm{CBD} /$ placebo administration. The

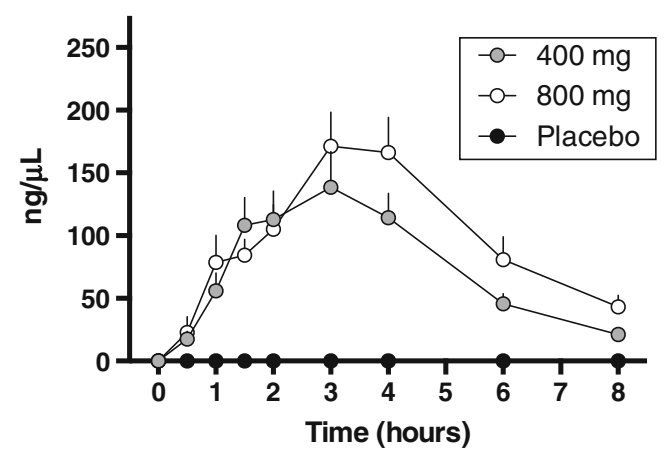

Fig. 5 Time course of plasma concentrations of $400 \mathrm{mg}$ and $800 \mathrm{mg}$ cannabidiol and placebo in combination with a potent opioid fentanyl in healthy individuals. Modified from Manini et al. [82] a

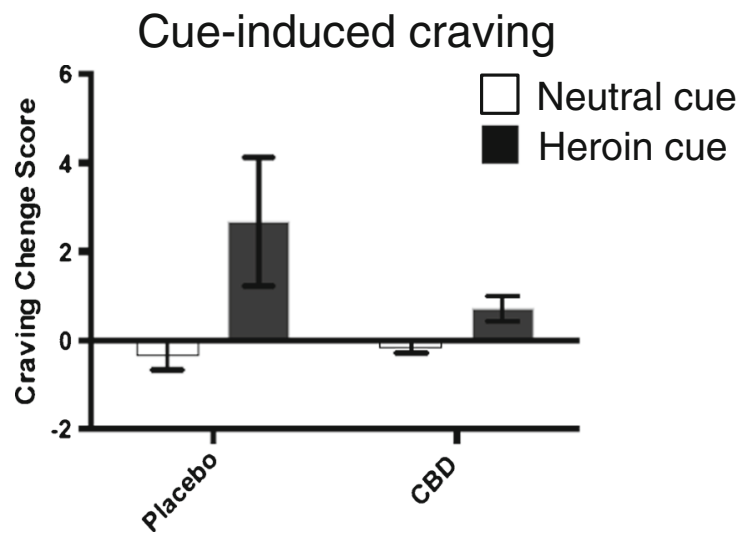

b

General craving difference from baseline
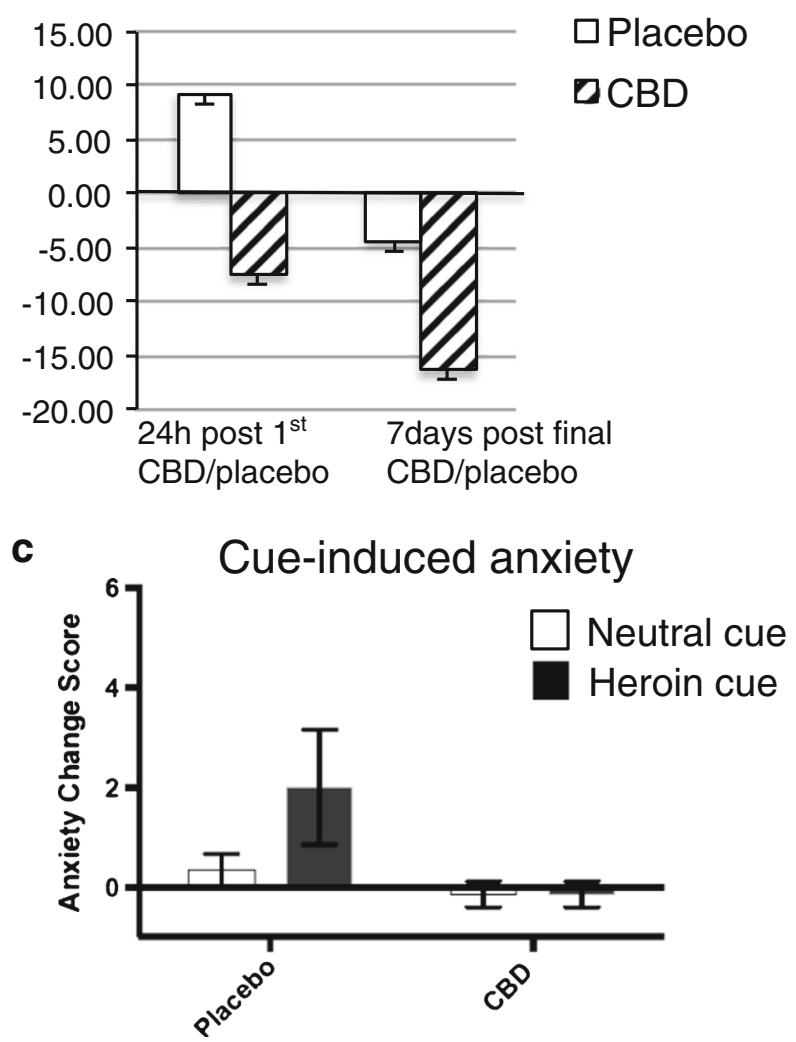

Fig. 6 The effect of cannabidiol (CBD) on craving and anxiety in abstinent heroin-dependent subjects. (a) Cue-induced craving (visual analog scale; VAS) induced by heroin video cue was blunted by a single administration of CBD (400 $\mathrm{mg}$ or $800 \mathrm{mg}$ combined) in comparison with placebo. Craving calculated as the change scores between pre- and postexposure to neutral or heroin cue videos. (b) General craving (heroin craving questionnaire) tended to decrease $24 \mathrm{~h}$ after a single administration of CBD that remained 7 days after 3 daily administrations of CBD in comparison with placebo. Craving calculated as change scores from pre-CBD administration (session 1) score. (c) Cue-induced anxiety induced by heroin cue was blunted $1 \mathrm{~h}$ after a single administration of CBD (400 mg or $800 \mathrm{mg}$ combined) in comparison with placebo. Anxiety calculated as the change scores between pre- and postexposure of neutral or heroin cue videos $(n=3-6)$. Data are mean $\pm \mathrm{SD}$ 
results showed that a single administration of $\mathrm{CBD}$, in comparison to placebo, attenuated subjective cue-induced craving measured after $1 \mathrm{~h}$ using the VAS craving scale (Fig. 6a). The single administration of CBD even maintained a decrease of general craving $24 \mathrm{~h}$ later, assessed using a heroin-craving questionnaire (Fig. 6b). Furthermore, the effect of CBD in reducing craving persisted even 7 days after the last treatment. Interestingly, the effects of CBD were also evident on anxiety in which there was an effective reduction in VAS anxiety measures (Fig. 6c). These preliminary pilot human study findings support the preclinical evidence and clinical studies that have evaluated anxiety, suggesting a potential therapeutic efficacy of CBD to reduce negative states in opioid-dependent individuals, which may, in turn, predict reduced craving and hence reduce the likelihood of relapse behavior.

An investigation with a larger number of participants is currently being conducted, but it is clear more studies are necessary to confirm these preliminary findings, as well as to evaluate different treatment schedules in order to fully evaluate the spectrum of CBD's effects. Nevertheless, the current human data are consistent with results from the rat models, suggesting that CBD attenuates cue-induced and general craving in opioid-dependent individuals and that the effects are protracted even after the acute exposure to the cannabinoid [62-64]. Overall, the pilot human and preclinical animal laboratory studies provide a foundation for continued exploration of CBD in treating opioid dependence.

\section{Conclusion}

Despite its long history of pervasive recreational use in society, the understanding of medicinal aspects of cannabinoids is only in its infancy. Significant research efforts are still necessary to evaluate fully the development of CBD as a potential therapy for addiction disorders. To date, the evidence appears to at least support a potential beneficial treatment for opioid abuse. The fact that patients with substance use disorders often present with various psychiatric and medical symptoms that are reduced by CBD - symptoms such as anxiety, mood symptoms, insomnia, and pain - also suggests that CBD might be beneficial for treating opioid-dependent individuals. Currently most medications for opioid abuse directly target the endogenous opioid system. CBD could thus offer a novel line of research medication that indirectly regulate neural systems modulating opioid-related behavior, thus helping to reduce side effects normally associated with current opioid substitution treatment strategies.

The fact that CBD and THC have divergent effects on behaviors linked to addiction vulnerability emphasizes the important need to educate the general public. Medical marijuana represents a complex chemical mixture, all of which may not be an appropriate treatment for substance use disorders; while one cannabinoid constituent in the plant can alleviate negative symptoms, another may exacerbate them. As such, it is important to make a distinction in the nomenclature and emphasize that it is specific cannabinoids, such as "CBD", that may hold the psychiatric therapeutic promise, not the general marijuana plant. As more research efforts are directed towards cannabinoids, we will soon be able to understand how best to leverage the potentially beneficial properties of cannabinoids to develop more targeted treatment interventions.

Acknowledgments This work was supported by grants from the National Institutes of Health grant DA027781 (YLH) and CTSA (UL1RR029887) at Mount Sinai Hospital. D.J.A. holds a clinical researcher career award from the Fonds de la Recherche en Santé du Québec. We thank GW Pharmaceutical for the cannabidiol used in our human studies and the National Institute on Drug Abuse's Drug Supply Program for the cannabidiol used in the animal studies.

Required Author Forms Disclosure forms provided by the authors are available with the online version of this article.

\section{References}

1. Elsohly MA, Slade D. Chemical constituents of marijuana: the complex mixture of natural cannabinoids. Life Sci 2005;78:539548.

2. Mechoulam R, Peters M, Murillo-Rodriguez E, Hanus LO. Cannabidiol—recent advances. Chem Biodivers 2007;4:1678-1692.

3. Mehmedic Z, Chandra S, Slade D, et al. Potency trends of $\Delta 9$-THC and other cannabinoids in confiscated cannabis preparations from 1993 to 2008. J Forensic Sci 2010;55:1209-1217.

4. Cilio MR, Thiele EA, Devinsky O. The case for assessing cannabidiol in epilepsy. Epilepsia 2014;55:787-790.

5. Devinsky O, Cilio MR, Cross H, et al. Cannabidiol: pharmacology and potential therapeutic role in epilepsy and other neuropsychiatric disorders. Epilepsia 2014;55:791-802.

6. Nestler EJ. The neurobiology of cocaine addiction. Sci Pract Perspect 2005;3:4-10.

7. Galanter M, Kleber HD. The American Psychiatric Publishing textbook of substance abuse treatment. 4th ed. American Psychiatric Publishing, Washington, DC, 2008.

8. Gossop M, Marsden J, Stewart D, Kidd T. The National Treatment Outcome Research Study (NTORS): 4-5 year follow-up results. Addiction 2003;98:291-303.

9. Rohsenow DJ, Martin RA, Eaton CA, Monti PM. Cocaine craving as a predictor of treatment attrition and outcomes after residential treatment for cocaine dependence. J Stud Alcohol Drugs 2007;68: 641-648.

10. Epstein DH, Preston KL. Daily life hour by hour, with and without cocaine: an ecological momentary assessment study. Psychopharmacology 2010;211:223-232.

11. Fox HC, Hong KI, Siedlarz K, Sinha R. Enhanced sensitivity to stress and drug/alcohol craving in abstinent cocaine-dependent individuals compared to social drinkers. Neuropsychopharmacology 2008;33:796-805.

12. Paliwal P, Hyman SM, Sinha R. Craving predicts time to cocaine relapse: further validation of the Now and Brief versions of the cocaine craving questionnaire. Drug Alcohol Depend 2008;93: $252-259$. 
13. Epstein DH, Willner-Reid J, Vahabzadeh M, et al. Real-time electronic diary reports of cue exposure and mood in the hours before cocaine and heroin craving and use. Arch Gen Psychiatry 2009;66: 88-94.

14. Preston KL, Vahabzadeh M, Schmittner J, et al. Cocaine craving and use during daily life. Psychopharmacology (Berl) 2009;207: 291-301.

15. Sinha R, Garcia M, Paliwal P, Kreek MJ, Rounsaville BJ. Stressinduced cocaine craving and hypothalamic-pituitary-adrenal responses are predictive of cocaine relapse outcomes. Arch Gen Psychiatry 2006;63:324-331.

16. Knapp WP, Soares BG, Farrel M, Lima MS. Psychosocial interventions for cocaine and psychostimulant amphetamines related disorders. Cochrane Database Syst Rev 2007:CD003023.

17. Amato L, Minozzi S, Pani PP, Davoli M. Antipsychotic medications for cocaine dependence. Cochrane Database Syst Rev 2007: CD006306.

18. Lima MS, Reisser AA, Soares BG, Farrell M. Antidepressants for cocaine dependence. Cochrane Database Syst Rev 2003: CD002950.

19. Minozzi S, Amato L, Davoli M, et al. Anticonvulsants for cocaine dependence. Cochrane Database Syst Rev 2008:CD006754.

20. Kosten TR, Domingo CB, Shorter D, et al. Vaccine for cocaine dependence: a randomized double-blind placebo-controlled efficacy trial. Drug Alcohol Depend 2014;140:42-47.

21. Alving CR, Matyas GR, Torres O, Jalah R, Beck Z. Adjuvants for vaccines to drugs of abuse and addiction. Vaccine 2014;32:53825389.

22. Rodriguez JJ, Mackie K, Pickel VM. Ultrastructural localization of the $\mathrm{CB} 1$ cannabinoid receptor in mu- opioid receptor patches of the rat Caudate putamen nucleus. J Neurosci 2001;21:823-833.

23. Zhang HY, Gao M, Liu QR, et al. Cannabinoid CB2 receptors modulate midbrain dopamine neuronal activity and dopaminerelated behavior in mice. Proc Natl Acad Sci U S A 2014;111: E5007-E5015.

24. Xi ZX, Peng XQ, Li X, et al. Brain cannabinoid CB(2) receptors modulate cocaine's actions in mice. Nat Neurosci 2011;14:1160 1166.

25. Pertwee RG. The diverse $\mathrm{CB} 1$ and $\mathrm{CB} 2$ receptor pharmacology of three plant cannabinoids: delta9-tetrahydrocannabinol, cannabidiol and delta9-tetrahydrocannabivarin. Br J Pharmacol 2008;153:199_ 215.

26. Thomas A, Baillie GL, Phillips AM, et al. Cannabidiol displays unexpectedly high potency as an antagonist of $\mathrm{CB} 1$ and $\mathrm{CB} 2$ receptor agonists in vitro. Br J Pharmacol 2007;150:613-623.

27. Qin N, Neeper MP, Liu Y, et al. TRPV2 is activated by cannabidiol and mediates CGRP release in cultured rat dorsal root ganglion neurons. J Neurosci 2008;28:6231-6238.

28. Bisogno T, Hanus L, De Petrocellis L, et al. Molecular targets for cannabidiol and its synthetic analogues: effect on vanilloid VR1 receptors and on the cellular uptake and enzymatic hydrolysis of anandamide. Br J Pharmacol 2001;134:845-852.

29. Campos AC, Guimaraes FS. Involvement of 5HT1A receptors in the anxiolytic-like effects of cannabidiol injected into the dorsolateral periaqueductal gray of rats. Psychopharmacology 2008;199: 223-230.

30. Magen I, Avraham Y, Ackerman Z, et al. Cannabidiol ameliorates cognitive and motor impairments in bile-duct ligated mice via 5HT1A receptor activation. Br J Pharmacol 2010;159:950-957.

31. Russo EB, Burnett A, Hall B, Parker KK. Agonistic properties of cannabidiol at 5-HT1a receptors. Neurochem Res 2005;30:10371043 .

32. Alves FH, Crestani CC, Gomes FV, et al. Cannabidiol injected into the bed nucleus of the stria terminalis modulates baroreflex activity through 5-HT1A receptors. Pharmacol Res 2010;62:228-236.
33. Gomes FV, Reis DG, Alves FH, et al. Cannabidiol injected into the bed nucleus of the stria terminalis reduces the expression of contextual fear conditioning via 5-HT1A receptors. J Psychopharmacol 2012;26:104-113.

34. Soares Vde P, Campos AC, Bortoli VC, et al. Intra-dorsal periaqueductal gray administration of cannabidiol blocks paniclike response by activating 5-HT1A receptors. Behav Brain Res 2010;213:225-229.

35. Zanelati TV, Biojone C, Moreira FA, Guimaraes FS, Joca SR Antidepressant-like effects of cannabidiol in mice: possible involvement of 5-HT1A receptors. Br J Pharmacol 2010;159:122128.

36. Pandolfo P, Silveirinha V, dos Santos-Rodrigues A, et al. Cannabinoids inhibit the synaptic uptake of adenosine and dopamine in the rat and mouse striatum. Eur J Pharmacol 2011;655:38 45.

37. Kathmann M, Flau K, Redmer A, Trankle C, Schlicker E. Cannabidiol is an allosteric modulator at mu- and delta-opioid receptors. Naunyn Schmiedebergs Arch Pharmacol 2006;372:354361

38. Carrier EJ, Auchampach JA, Hillard CJ. Inhibition of an equilibrative nucleoside transporter by cannabidiol: a mechanism of cannabinoid immunosuppression. Proc Natl Acad Sci U S A 2006;103: 7895-7900.

39. Hampson AJ, Grimaldi M, Axelrod J, Wink D. Cannabidiol and (-)Delta9-tetrahydrocannabinol are neuroprotective antioxidants. Proc Natl Acad Sci U S A 1998;95:8268-8273.

40. Hallak JE, Dursun SM, Bosi DC, et al. The interplay of cannabinoid and NMDA glutamate receptor systems in humans: preliminary evidence of interactive effects of cannabidiol and ketamine in healthy human subjects. Prog Neuropsychopharmacol Biol Psychiatry 2011;35:198-202.

41. Guimaraes FS, Chiaretti TM, Graeff FG, Zuardi AW. Antianxiety effect of cannabidiol in the elevated plus-maze. Psychopharmacology 1990;100:558-559.

42. Guimaraes FS, de Aguiar JC, Mechoulam R, Breuer A. Anxiolytic effect of cannabidiol derivatives in the elevated plus-maze. Gen Pharmacol 1994;25:161-164.

43. Moreira FA, Aguiar DC, Guimaraes FS. Anxiolytic-like effect of cannabidiol in the rat Vogel conflict test. Prog Neuropsychopharmacol Biol Psychiatry 2006;30:1466-1471.

44. Granjeiro EM, Gomes FV, Guimaraes FS, Correa FM, Resstel LB. Effects of intracisternal administration of cannabidiol on the cardiovascular and behavioral responses to acute restraint stress. Pharmacol Biochem Behav 2011;99:743-748.

45. Uribe-Marino A, Francisco A, Castiblanco-Urbina MA, et al. Antiaversive effects of cannabidiol on innate fear-induced behaviors evoked by an ethological model of panic attacks based on a prey vs the wild snake Epicrates cenchria crassus confrontation paradigm. Neuropsychopharmacology 2012;37:412-421.

46. Fagherazzi EV, Garcia VA, Maurmann N, et al. Memory-rescuing effects of cannabidiol in an animal model of cognitive impairment relevant to neurodegenerative disorders. Psychopharmacology (Berl) 2012;219:1133-1140.

47. Avraham Y, Grigoriadis N, Poutahidis T, et al. Cannabidiol improves brain and liver function in a fulminant hepatic failureinduced model of hepatic encephalopathy in mice. Br J Pharmacol 2011;162:1650-1658

48. Cassol-Jr OJ, Comim CM, Silva BR, et al. Treatment with cannabidiol reverses oxidative stress parameters, cognitive impairment and mortality in rats submitted to sepsis by cecal ligation and puncture. Brain Res 2010;1348:128-138.

49. El-Alfy AT, Ivey K, Robinson K, et al. Antidepressant-like effect of delta9-tetrahydrocannabinol and other cannabinoids isolated from Cannabis sativa L. Pharmacol Biochem Behav 2010;95:434-442. 
50. Deiana S, Watanabe A, Yamasaki Y, et al. Plasma and brain pharmacokinetic profile of cannabidiol (CBD), cannabidivarine (CBDV), Delta(9)-tetrahydrocannabivarin (THCV) and cannabigerol $(\mathrm{CBG})$ in rats and mice following oral and intraperitoneal administration and CBD action on obsessive-compulsive behaviour. Psychopharmacology (Berl) 2012;219:859-873.

51. Casarotto PC, Gomes FV, Resstel LB, Guimaraes FS. Cannabidiol inhibitory effect on marble-burying behaviour: involvement of CB1 receptors. Behav Pharmacol 2010;21:353-358.

52. Bornheim LM. Effect of cytochrome P450 inducers on cocainemediated hepatotoxicity. Toxicol Appl Pharmacol 1998;150:158-165.

53. Hamelink C, Hampson A, Wink DA, Eiden LE, Eskay RL. Comparison of cannabidiol, antioxidants, and diuretics in reversing binge ethanol-induced neurotoxicity. J Pharmacol Exp Ther 2005;314:780-788.

54. Nahas G, Trouve R. Effects and interactions of natural cannabinoids on the isolated heart. Proc Soc Exp Biol Med 1985;180:312-316.

55. Trouve R, Nahas G, Stuker O, Latour C. [Antagonistic effects of two natural cannabinoids on the isolated heart]. C R Seances Acad Sci III 1983;297:191-194 (in Frecnh).

56. Long LE, Chesworth R, Huang XF, et al. A behavioural comparison of acute and chronic Delta9-tetrahydrocannabinol and cannabidiol in C57BL/6JArc mice. Int J Neuropsychopharmacol 2010;13:861-876.

57. Crippa JA, Zuardi AW, Garrido GE, et al. Effects of cannabidiol (CBD) on regional cerebral blood flow. Neuropsychopharmacology 2004;29:417-426.

58. Zuardi AW, Hallak JE, Dursun SM, et al. Cannabidiol monotherapy for treatment-resistant schizophrenia. J Psychopharmacol 2006;20: 683-686.

59. Zuardi A, Crippa J, Dursun S, et al. Cannabidiol was ineffective for manic episode of bipolar affective disorder. J Psychopharmacol 2010;24:135-137.

60. Zuardi AW, Morais SL, Guimaraes FS, Mechoulam R. Antipsychotic effect of cannabidiol. J Clin Psychiatry 1995;56: 485-486.

61. Iseger TA, Bossong MG. A systematic review of the antipsychotic properties of cannabidiol in humans. Schizophr Res 2015;162:153161.

62. Crippa JA, Derenusson GN, Ferrari TB, et al. Neural basis of anxiolytic effects of cannabidiol (CBD) in generalized social anxiety disorder: a preliminary report. J Psychopharmacol 2011;25:121-130.

63. Zuardi AW, Shirakawa I, Finkelfarb E, Karniol IG. Action of cannabidiol on the anxiety and other effects produced by delta 9THC in normal subjects. Psychopharmacology 1982;76:245-250.

64. Bergamaschi MM, Queiroz RH, Chagas MH, et al. Cannabidiol reduces the anxiety induced by simulated public speaking in treatmentnaive social phobia patients. Neuropsychopharmacology 2011;36: 1219-1226.

65. Fusar-Poli P, Crippa JA, Bhattacharyya S, et al. Distinct effects of \{delta\}9-tetrahydrocannabinol and cannabidiol on neural activation during emotional processing. Arch Gen Psychiatry 2009;66:95-105.

66. Schier AR, Ribeiro NP, Silva AC, et al. Cannabidiol, a Cannabis sativa constituent, as an anxiolytic drug. Rev Bras Psiquiatr 2012;34(Suppl. 1):S104-S110.

67. LaLumiere RT, Kalivas PW. Glutamate release in the nucleus accumbens core is necessary for heroin seeking. J Neurosci 2008;28: $3170-3177$.
68. Katona I, Freund TF. Endocannabinoid signaling as a synaptic circuit breaker in neurological disease. Nat Med 2008;14:923-930.

69. Parker LA, Burton P, Sorge RE, Yakiwchuk C, Mechoulam R. Effect of low doses of delta9-tetrahydrocannabinol and cannabidiol on the extinction of cocaine-induced and amphetamine-induced conditioned place preference learning in rats. Psychopharmacology (Berl) 2004;175:360-366.

70. Vann RE, Gamage TF, Warner JA, et al. Divergent effects of cannabidiol on the discriminative stimulus and place conditioning effects of Delta(9)-tetrahydrocannabinol. Drug Alcohol Depend 2008;94:191-198.

71. Katsidoni V, Anagnostou I, Panagis G. Cannabidiol inhibits the reward-facilitating effect of morphine: involvement of 5-HT1A receptors in the dorsal raphe nucleus. Addict Biol 2013;18:286-296.

72. Ren Y, Whittard J, Higuera-Matas A, Morris CV, Hurd YL. Cannabidiol, a nonpsychotropic component of cannabis, inhibits cue-induced heroin seeking and normalizes discrete mesolimbic neuronal disturbances. J Neurosci 2009;29:14764-14769.

73. Knackstedt LA, Kalivas PW. Glutamate and reinstatement. Curr Opin Pharmacol 2009;9:59-64.

74. Hine B, Torrelio M, Gershon S. Interactions between cannabidiol and delta9-THC during abstinence in morphine-dependent rats. Life Sci 1975;17:851-857.

75. Hine B, Torrelio M, Gershon S. Differential effect of cannabinol and cannabidiol on THC-induced responses during abstinence in morphine-dependent rats. Res Commun Chem Pathol Pharmacol 1975;12:185-188.

76. Chesher GB, Jackson DM. The quasi-morphine withdrawal syndrome: effect of cannabinol, cannabidiol and tetrahydrocannabinol. Pharmacol Biochem Behav 1985;23:13-15.

77. Bhargava HN. Effect of some cannabinoids on naloxoneprecipitated abstinence in morphine-dependent mice. Psychopharmacology 1976;49:267-270.

78. Klein C, Karanges E, Spiro A, et al. Cannabidiol potentiates Delta(9)-tetrahydrocannabinol (THC) behavioural effects and alters THC pharmacokinetics during acute and chronic treatment in adolescent rats. Psychopharmacology (Berl) 2011;218:443-457.

79. Morgan CJ, Das RK, Joye A, Curran HV, Kamboj SK. Cannabidiol reduces cigarette consumption in tobacco smokers: preliminary findings. Addict Behav 2013;38:2433-2436.

80. Morgan CJ, Schafer G, Freeman TP, Curran HV. Impact of cannabidiol on the acute memory and psychotomimetic effects of smoked cannabis: naturalistic study: naturalistic study [corrected]. Br J Psychiatry 2010;197:285-290.

81. Morgan CJ, Freeman TP, Schafer GL, Curran HV. Cannabidiol attenuates the appetitive effects of Delta 9-tetrahydrocannabinol in humans smoking their chosen cannabis. Neuropsychopharmacology 2010;35:1879-1885.

82. Manini AF, Yiannoulos G, Bergamaschi MM, et al. Safety and pharmacokinetics of oral cannabidiol when administered concomitantly with intravenous fentanyl in humans. J Addict Med 2015;9: 204-210.

83. Fusar-Poli P, Allen P, Bhattacharyya S, et al. Modulation of effective connectivity during emotional processing by Delta 9tetrahydrocannabinol and cannabidiol. Int J Neuropsychopharmacol 2010;13:421-432. 